THE JOURNAL OF

THOBAGIG AND

GARDIOVASGULAR

SURGERY

Vol. 135, No. 4, April 2008

\title{
The surgical treatment for atrial fibrillation: A call for standardization
}

Niv Ad, MD

See related article on page 863.
From the Inova Heart and Vascular Institute, Falls Church, Va.

Niv Ad reports consulting fees from Atricure, Estech, and Boston Scientific and lecture fees from Medtronic.

Received for publication Sept 15, 2007; accepted for publication Oct 2, 2007.

Address for reprints: Niv Ad, MD, Inova Heart and Vascular Institute, 3300 Gallows Rd, Falls Church, VA 22042 (E-mail: nivadmd@hotmail.com).

J Thorac Cardiovasc Surg 2008;135:727-8 $0022-5223 / \$ 34.00$

Copyright (C) 2008 by The American Association for Thoracic Surgery

doi:10.1016/j.jtcvs.2007.10.037
$\mathrm{I}$ $\mathrm{n}$ recent years, there has been a significant increase in the number of surgical procedures performed to ablate atrial fibrillation. As a result, a large number of publications detailing subsequent outcomes after such procedures have resulted. However, most reports do not address the true effect of the procedure on patient outcomes and well-being. Second, an additional dilemma regarding the synthesis of literature addressing surgical ablation is the lack of standardization. Patients are being treated with different ablation tools and energy sources and different lesion sets, and the follow-up with subsequent reporting fails to address the difficulties of comparisons between the programs. The literature also lacks information regarding the correlation between the success in ablating atrial fibrillation and favorable long-term outcomes. In an attempt to standardize the reports and improve our understanding as to the real effect of surgical ablation, Dr Melo established the International Registry for Atrial Fibrillation.

In this issue of the Journal, Melo and colleagues ${ }^{1}$ discuss 5 -year results from the International Registry for Atrial Fibrillation. After analysis of more than 1700 patients operated on for mitral valve disease and atrial fibrillation from participating institutions in Europe and the United States, the authors conclude that achievement of stable sinus rhythm is an excellent predictor for better survival and a decreased incidence of thromboembolic events. Among the predictors of better survival were smaller left atrial size, biatrial ablation procedures, the absence of concomitant coronary artery bypass grafting, and the lack of preoperative permanent atrial fibrillation.

Registries $^{2}$ generally exist to describe the natural history of disease in a nonrandomized clinical trial setting, pool a large number of similar cases for analyses that would otherwise be restricted to smaller samples, and provide real-world comparisons of cost, cost-effectiveness, and patient outcome for new treatment modalities. They should also provide estimates of long-term follow-up. Registries can be classified according to the population that was defined. For example, phase IV postmarketing studies sponsored by the pharmaceutical industry are registries. Health service registries can consist of patients aggregated by a common procedure, disease, institution, or even insurance payer. There are several key steps in planning a patient registry, including clearly defining the purpose of the registry, determining whether this is the appropriate tool to address the research question, recognizing the stakeholders, delivering a clear definition of the scope and the target population, assessing the feasibility, and securing the funding. Furthermore, the creation of a registry presents unique and challenging methodological issues. How are various surgical protocols reconciled and aggregated for analysis? With what data are reconciliations made and by whom? For every new research hypothesis, will data aggregation strategies be revisited? Only so much clinical information can be collected by participating institutions before the benefits of participating are outweighed by the negatives. Additionally, how does the registry cope with data from multiple 
institutions with multiple protocols? Standardization is very difficult, and if standardization is applied, are all cases before the registry start date exempt from analysis?

From a statistical viewpoint, the analysis of registry data presents several issues worth noting. In a randomized clinical trial the randomization process equally distributes potential confounding variables among the study arms. During subsequent analysis, for reasons beyond the scope of this article, unless a blocking methodology is used to ensure a constant, equally distributed distribution of potential confounders, the analyst need not waste resources (ie, degrees of freedom) on needless variables during the modeling process because they have already been accounted for in the randomization scheme. Unfortunately, this is not the case when analyzing registry data containing multiple institutions with multiple protocols. In effect, these institutions represent the blocks that statisticians designing randomized clinical trials so painstakingly try to achieve. Therefore every analysis of registry data should begin with a lengthy exploration of the effects of institution and protocol on the relationships between suspected predictors and the adverse outcomes under scrutiny. If no effect is observed, then these parameters can be discarded, and the real analysis can begin.

But what if institutional effects are observed during the analysis? Although analyses with large samples will undoubtedly reveal differences between small- and large-volume institutions, statistical control is readily available. Furthermore, what about the interpretation of data? Should interactive effects be explored between institutions? Should they even be reported? The analyst is faced with the dilemma of statistical parsimony or oversaturation. Parsimony is simply achieving the most efficient statistical analysis by using the fewest resources. Oversaturation is the phenomenon of overmodeling the data such that the used statistical algorithms collapse and generate meaningless or even no results. Registries that serve as observational studies are wonderful and useful tools in the gathering of knowledge but require difficult and careful analytic methodologies. Lastly, at first glance, one might wonder why authorship would pose any issue at all. However, the initial question to be asked is who owns the data. Obviously, the founders of the registry have a vested interest in receiving credit for any published work and rightfully so, but are the data owned by the registry founders or the participating members?

The study of Melo and colleagues ${ }^{1}$ published in this issue reports 5-year results from a large cohort of patients with mitral valve disease and atrial fibrillation. The authors should be congratulated for their contribution in establishing such a large pool of data. But we have to ask ourselves the following: What is the real contribution of such registry? The contribution of greatest value of such a registry is epidemiologic in nature because the study provides us with valuable information regarding the number and type of procedures performed in various centers in Europe and the United States. The centers and the surgeons are well established in the field, and the following issues are not with their surgical expertise. The regis- try also provides us with information regarding the extent of different ablative energy source use. However, can we really draw any conclusion regarding clinical outcomes? There are 10 different centers with various patient-management protocols. The authors clearly state that the study was designed to assess the clinical and survival benefits of sinus rhythm recovery in this subset of patients. The report also includes short- and long-term results detailing survival and the use of antiarrhythmic therapy with data collected retrospectively.

The registry is composed of a large pool of patients for which 56 different clinical variables were collected retrospectively, with no report regarding the protocol to treat missing data. The authors freely admit that centers were allowed to use their own protocols, resulting in different surgical approaches, including lesion set, ablation technology, and individual definitions regarding follow-up. As a result, no standardization of the preoperative assessment, intraoperative management, and postoperative care was established. Surgeons were allowed to treat atrial fibrillation surgically according to their own philosophy without any control. The postoperative management was not the same for the different centers, rhythm follow-up was accomplished according to some rules, and not all the patients have been followed by using the same tools (some had repeated electrocardiographic analysis, some had Holter monitoring, and some had a combination). No guidelines were established with regard to antiarrhythmic and anticoagulation treatment (and we know well that some patients still take the medication without any indications for various reasons). These potential biases and problems are not unique to this registry alone. As mentioned earlier, creating a registry poses a challenge on multiple fronts. This study is a very good example of the challenges we are facing in the field of the surgical treatment of atrial fibrillation.

The work of Melo and colleagues ${ }^{1}$ in this issue epitomizes the current dilemma in assessing the real effect of the surgical treatment of atrial fibrillation, namely the complete lack of credibility for the outstanding successes after the maze procedure and its modifications. The newly published Heart Rhythm Society guidelines ${ }^{3}$ can serve as a good foundation to address the steps to establish standardized approaches in this growing field. We should actively pursue and adopt standardized definitions for the collection of prospective data to allow for solid conclusions in the important field of the surgical treatment for atrial fibrillation.

I thank Scott. D. Barnett, PhD.

\section{References}

1. Melo J, Santiago T, Aguiar C, Berglin E, Knaut M, Alfieri O, et al. Surgery for atrial fibrillation in mitral patients. Results at five years from the International Registry of Atrial Fibrillation. J Thorac Cardiovasc Surg. 2008;135:863-9.

2. Rothman K, Greenland S. Modern epidemiology. Beverly Farms (Mass): OEM Press; 1998.

3. Heart Rhythm Society. Consensus statement on catheter and surgical ablation for AF. Available at: http://www.hrsonline.org/News/Media/ press-releases/CSAblation.cfm. Accessed December 10, 2007. 